\title{
Imaging in Cataract Surgery
}

\author{
Mahmood Khan ${ }^{1}$ and Zaina Al-Mohtaseb² \\ 1. Department of Ophthalmology, Weill Cornell Medical College, New York, NY, USA; 2. Cullen Eye Institute, Department of Ophthalmology, \\ Baylor College of Medicine, Houston, TX, USA
}

DOI: https://doi.org/10.17925/EOR.2019.13.2.71

W ith increased patient expectations for precise post-operative refractive results, the need for accurate keratometry, biometry, and appropriate intraocular lens power formula selection is of paramount importance. The past decade has brought improvements in topography, biometry, and intraocular lens (IOL) calculations and the advent of intra-operative imaging and aberrometry has assisted in achieving more accurate post-surgical refractive outcomes. This review aims to provide an overview of imaging modalities used in the pre-operative and intra-operative setting in anterior segment cataract surgery. Intraoperative imaging can be used in: capsulorhexis centration, wound and astigmatic keratometry placement, IOL centration and toric alignment. Specifically, we aim to provide an overview on the Zeiss Callisto (Zeiss, Oberkochen, Germany) imaging system used in toric lens alignment, the Alcon Verion (Alcon, Fort Worth, Texas, USA) used in combination with the ORA System ${ }^{\text {TM }}$ (WaveTec Vision, Aliso Viejo, California, USA) for toric lens alignment and intraoperative aberrometry for lens selections, and the TrueVision 3D System (TrueVision 3D Surgical, Goleta, CA, USA) used in toric lens alignment. The utility of intraoperative imaging in correcting astigmatism and selecting IOL powers in routine cataract surgery remains an adjuvant to current pre-operative keratometry and biometry. Although studies have shown effectiveness of utilising intraoperative imaging in cataract surgery, there remains a need for larger retrospective studies that compare the accuracy of current IOL formulas versus intraoperative aberrometry in both normal and post-refractive surgery.

\section{Keywords}

Cataract, toric alignment,

intraoperative imaging, lens

Disclosures: Zaina Al-Mohtaseb is a financial consultant to Alcon, Johnson \& Johnson and Zeiss. Mahmood Khan has nothing to declare in relation to this article.

Review Process: Double-blind peer review.

Compliance with Ethics: This study involves a review of the literature and did not involve any studies with human or animal subjects performed by any of the authors. Informed consent was received to use the patient images included in this article.

Authorship: The named authors meet the International Committee of Medical Journal Editors (ICMJE) criteria for authorship of this manuscript, take responsibility for the integrity of the work as a whole, and have given final approval for the version to be published.

Received: 14 October 2019

Accepted: 9 December 2019

Citation: European Ophthalmic Review. 2019;13(2):71-5

Corresponding Author: Zaina Al-Mohtaseb,

Cullen Eye Institute, Department of Ophthalmology,

Baylor College of Medicine, 6565 Fannin St

Houston, TX 77030, US. E: zaina1225@gmail.com

Support: No funding was received in

the publication of this article.
Increased patient expectations for precise postoperative refractive results have driven continued improvements in intraocular lens (IOL) calculations and preoperative biometry. Utilisation of accurate keratometry and biometry, and appropriate IOL power formula selection with optimised lens constants, are paramount.' Over the past decade, improvements in topography, biometry and IOL calculations and the advent of intraoperative imaging and aberrometry, has assisted in achieving more accurate post-surgical refractive outcomes.

Accurate measurements of the optical parameters of the eye, lead to achieving a desired target refraction. Keratometry is the measurement of the corneal radius of curvature. The axial length consists of the anterior chamber depth, lens thickness, and vitreous chamber depth, and these measurements can often result in vastly different IOL powers. ${ }^{2}$ One of the challenges in preoperative planning for IOL implantation is obtaining accurate biometric data. ${ }^{1,2}$ It has become one of the most important steps in modern cataract surgery and with appropriate formula selection, optical axial length measurement, and optimisation of IOL constants, the majority of patients are able to achieve an outcome within $\pm 0.5 \mathrm{D}$ of target refraction. ${ }^{3.4}$

Sources of error include imprecise axial length measurements secondary to dense cataracts which may inhibit some keratometry and biometers from obtaining accurate measurements, though new machines are more accurate concerning this. ${ }^{5}$ Additionally, many keratometry devices do not measure the posterior corneal astigmatism, and this can often result in under or over estimation of total corneal astigmatism, especially in cases where a toric lens is implanted. Other sources of error include sub-optimal ocular surface conditions that prohibit accurate and consistent corneal power measurements such as ocular surface disease and epithelial basement membrane dystrophy. Furthermore, errors in toric axis marking can lead to inaccurate alignment and often result in suboptimal visual outcomes. Over the years, several image-guided modalities have been developed for accurate toric IOL alignment that can help in decreasing the subjectivity associated with manual marking. ${ }^{6-10}$

Wavefront aberrometry can be utilised to obtain preoperative measurements or to confirm them in the intraoperative setting. An intraoperative aberrometer uses wavefront measurement technology to measure the biometry of the eye during cataract surgery and the measurement is made after the crystalline lens has been removed. ${ }^{.}$The device then suggests an IOL power and or toric alignment based on the refraction of the aphakic eye. 
Figure 1: Manual marking technique used for toric lens alignment and limbal relaxing incision placement

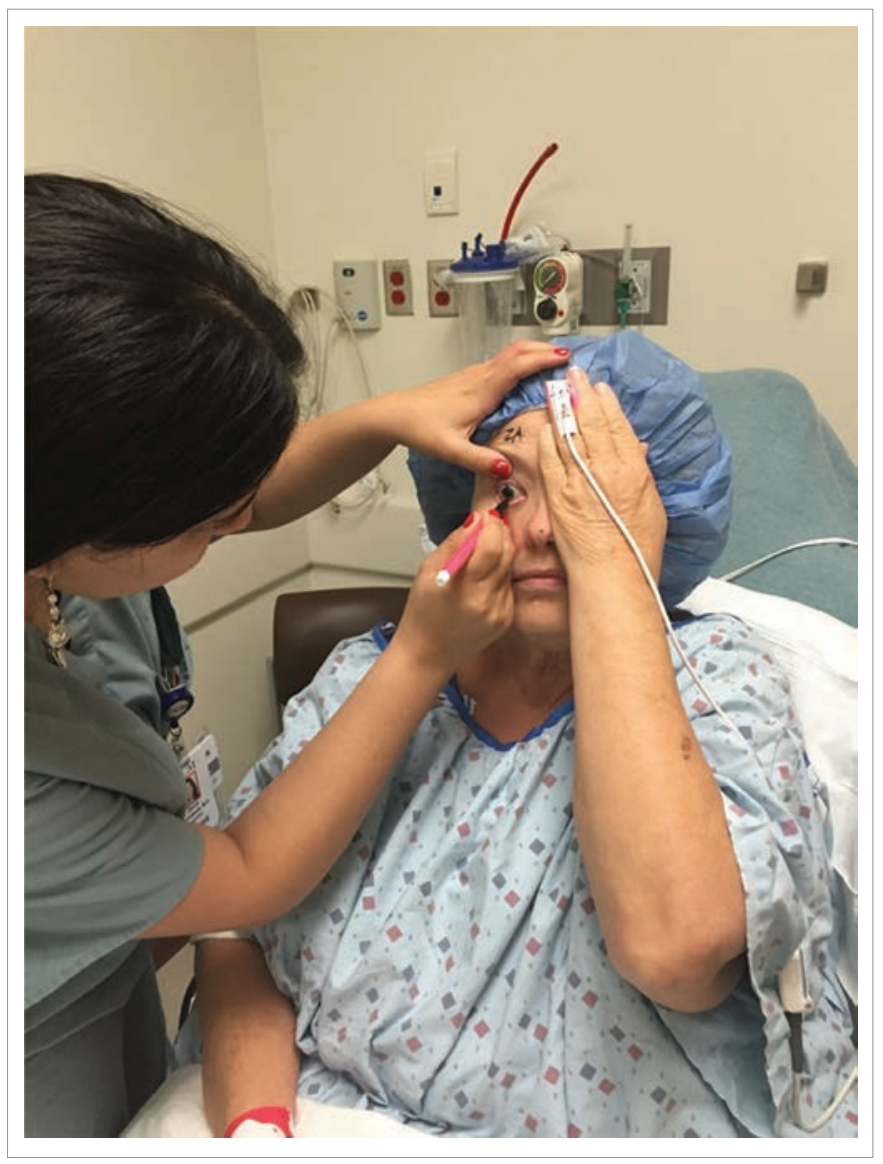

This review aims to provide an overview of imaging modalities used in the preoperative and intraoperative setting in anterior segment cataract surgery. Intraoperative imaging can be used in capsulorhexis centration, wound and astigmatic keratometry placement, IOL centration and toric alignment. Specifically, we aim to provide an overview on the Zeiss Callisto $^{\circledast}$ (Zeiss, Oberkochen, Germany) imaging system used in toric lens alignment, the Alcon Verion ${ }^{\mathrm{TM}}$ (Alcon, Fort Worth, TX, USA) used in combination with the ORA System ${ }^{\mathrm{TM}}$ (WaveTec Vision, Aliso Viejo, CA, USA) for toric lens alignment and intraoperative aberrometry for lens selections, and the TrueVision ${ }^{\circledR}$ 3D System (TrueVision 3D Surgical, Goleta, CA, USA) used in toric lens alignment.

\section{Toric lens alignment}

Preoperative planning for toric IOL placement requires accurate measurement of the axis, magnitude, and regularity of corneal astigmatism. Manual (Figure 1), automated, and simulated keratometry are amongst the most common methods utilised; with automated keratometry being the most reproduceable and repeatable..$^{11,12}$ It is well established that refractive performance depends on preoperative measurement, good intraoperative alignment of the IOL and its stability in the bag during the postoperative period. The most common cause of post-refractive error following toric IOL placement is inaccurate preoperative measurements or alignment errors intraoperatively. Minor alignment errors can results in 3\% loss of intended astigmatic correction per degree of misalignment, with $30 \%$ of misalignment resulting in complete loss of the intended astigmatic correction. ${ }^{2}$

Aberrometry-guided alignment can serve as a data point in reconfirming preoperative measurements. This technology has the benefit of allowing
Figure 2: Zeiss Callisto ${ }^{\circledR}$ is a component of the Zeiss cataract suite

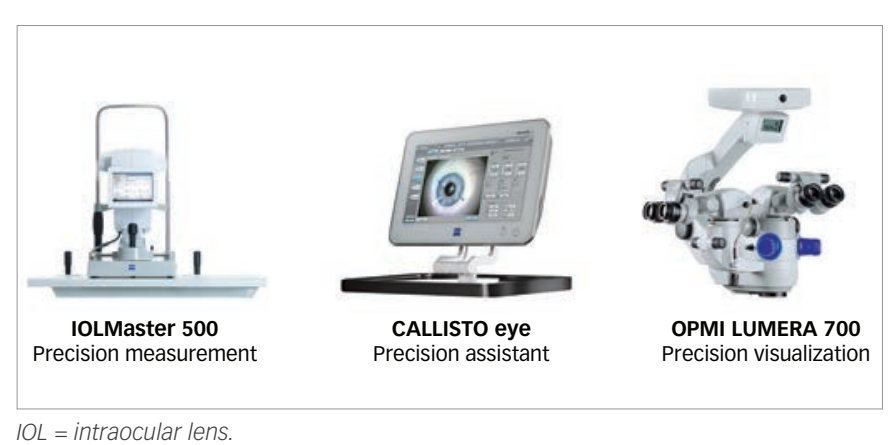

Figure 3: Alcon Verion ${ }^{\mathrm{TM}}$ System for used in intraoperative incisions, capsulotomies and intraocular-lens alignment

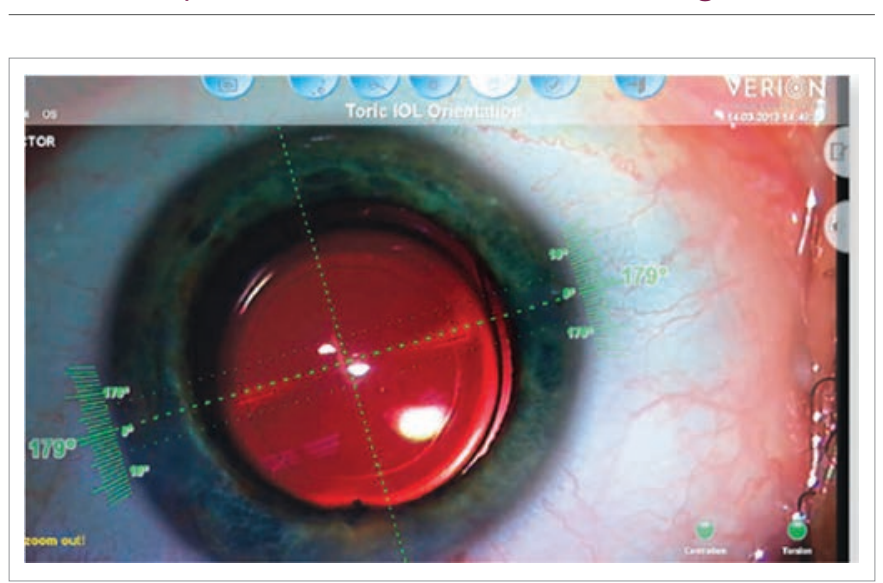

Photo courtesy of Lawrence Woodard, MD.

the surgeon to minimise refractive error by confirming the correct IOL power and axis of astigmatic error correction. ${ }^{13}$

\section{Zeiss Callisto}

The Zeiss Callisto (Figure 2) is a component of the Zeiss Cataract Suite which consists of the IOL Master, Callisto Eye, and OPMI Lumera 700 surgical Microscope. The IOL Master 500 and 700 obtain precise preoperative photographs that are matched to conjunctival markings and tracked intraoperatively, allowing for overlays of axis lines. Used in conjunction with the OPMI Lumera 700 Surgical Microscope, the Callisto Eye software allows for capsulorhexis centration, arcuate and main incision placement, multifocal IOL centration, and toric IOL alignment. In a study comparing manually marked versus Calisto Eye toric IOL alignment, deviation from the target axis of implantation and postoperative cylinder was statistically less in the latter. ${ }^{6}$ Tityal et al. compared manual marking with Zeiss Callisto-assisted toric IOL alignment and found that the deviation from target axis were $3.7 \pm 2.8$ degrees and $5.8 \pm 3.7$ degrees, respectively, at postoperative day $1, p<0.005$. Similarly, at postoperative day 30 , the deviation was $3.6 \pm 2.6$ degrees and $5.5 \pm 3.3$ degrees, respectively. ${ }^{6}$

\section{Alcon Verion}

The Alcon Verion System consists of the Verion Reference Unit and Verion Digital Marker that captures a reference image for use in intraoperative incisions, capsulotomies, and IOL alignment. This system utilises real-time intraoperative imaging to display astigmatic axis and anatomic landmarks for toric IOL alignment while compensating for eye movement, zoom, instruments and subconjunctival haemorrhage. Benefits of this system include its compatibility with platforms such as the LenS $x^{\circledR}$ (LenSX Laser Inc., Aliso Viejo, CA, USA) and most surgical microscopes. 
Figure 4: (A) TrueVision ${ }^{\circledR}$ 3D System intraoperative imaging overlay; (B) Cassini preoperative aberrometry
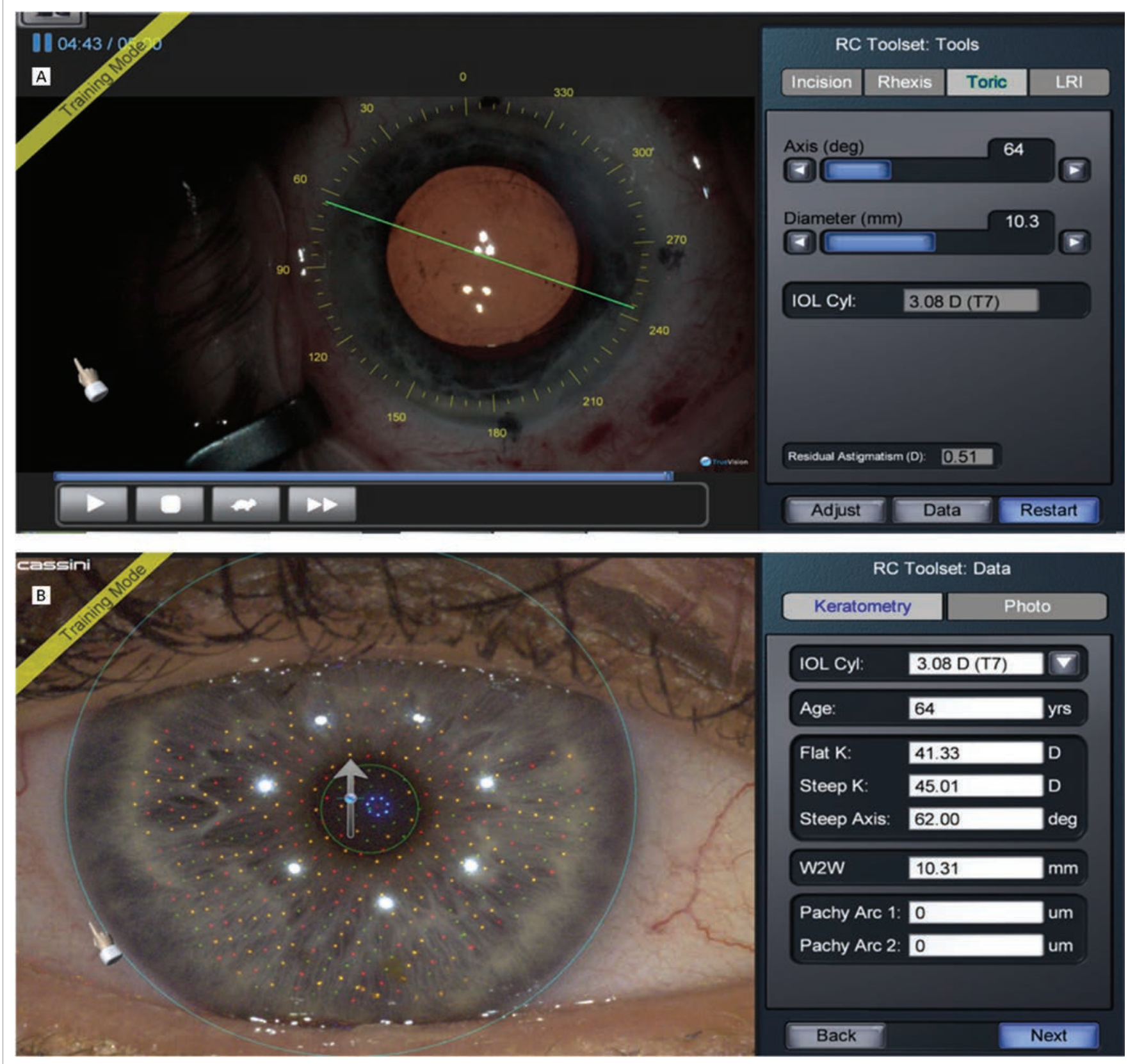

Similar to studies conducted on the Zeiss Callisto, a randomised prospective clinical study demonstrated that the Verion System resulted in less postoperative deviation from target induced astigmatism and showed less postoperative toric IOL misalignment than using a manual marking technique. The advantage of the Verion System lies in the integration of preoperative imaging consisting of a reference image, keratometry readings, preoperative planning of the surgery including toric IOL choice and incision site and lens power, and intraoperative guidance with an overlay line view. ${ }^{9}$ Elhofi et al. demonstrated that using the Alcon Verion system compared to traditional manual marking, resulted in a smaller mean toric misalignment at 1 hour ( $1.3 \pm 1.6$ versus $2.8 \pm 1.8, p=0.02)$ and 3 months postoperatively $(1.7 \pm 1.5$ versus $3.1 \pm 2.1, p<0.05)$, though this did not result in a clinically significant difference in uncorrected visual acuity. ${ }^{9}$

\section{TrueVision}

TrueVision's Visualization system displays the surgical field on a $3 \mathrm{D}$ flat panel display in the operation room and provides surgeons guidance in placing limbal relaxing incisions and toric IOL position to correct astigmatism. The system uses preoperative anterior segment photographs (Figure 4) to map images and project the steep axis throughout surgery displayed digitally in 3D. The ability of this suite to integrate with preoperative diagnostic devices, such as the Cassini (Cassini Technologies, The Hague, The Netherlands), Pentacam ${ }^{\circledast}$ (OCULUS, Wetzlar · Germany), OA-2000 (TOMEY, Nürnberg, Germany) and Lenstar (Haag-Streit, Koeniz, Switzerland) demonstrates an advantage over other systems that utilise preoperative imaging from within their respective companies. In a study examining TrueVision 3D compared with manual marking, it was found that 3D imaging error was -0.58 degrees \pm 3.90 standard deviation (SD) (range -9 to 5 degrees), and the mean manual ink error was $-0.27 \pm 3.65$ degrees (range -8 to 5 degrees), showing no statistical difference between TrueVision 3D and manual ink marking. ${ }^{13}$ Although the system is no longer commercially available, the 3D imaging technology portion of it is now owned by Alcon and marketed as the NGENUITY ${ }^{\circledR}$ 3D Visualization System for vitreoretinal surgery. 
Figure 5: The WaveTec ORA System ${ }^{\mathrm{TM}}$ intraoperative wave front aberrometer that allows for intraoperative refraction of phakic and pseudophakic eyes

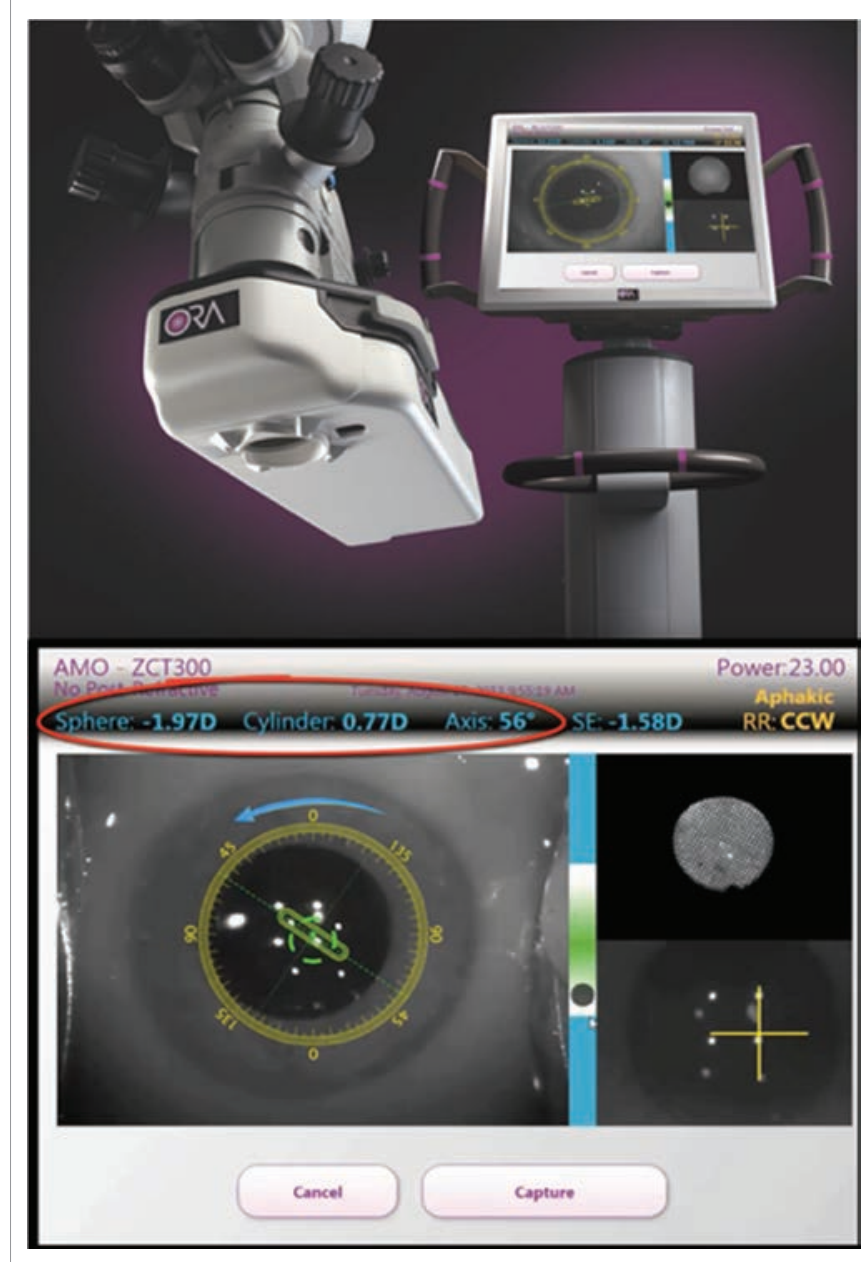

In a meta-analysis of toric IOL alignment using an image guided system (Verion, Callisto, and Truevision) versus manual marking by Zhou et al., the mean axis misalignment from pre- and postoperative measurements was $2.68 \pm 0.76 \mathrm{D}$ (142 eyes) and $4.06 \pm 1.08 \mathrm{D}$ (141 eyes), respectively. ${ }^{14}$

\section{Intraoperative wavefront aberrometry ORA System}

The ORA System (Figure 5) uses wavefront technology to analyse the optical pathway of the eye and assists the surgeon by helping them make the best choices for IOL power and astigmatism correction. It is attached to the surgical microscope and has a wide dioptric range for both aphakic and pseudophakic measurements that are taken in the operating room to guide IOL power selection and lens placement. ${ }^{15}$

In routine cataract surgery in the uncomplicated eye, there have been studies comparing intraoperative wavefront aberrometry to traditional formulas. Davison et al. aimed to assess the value of intraoperative aberrometry in determining IOL sphere power in eyes with no previous surgery. Lens type included multifocal IOLs and this study found that in $56 \%$ (35/63 eyes) of these cases, the intraoperative aberrometry result was the better option, and in $44 \%$ (28/63) of cases, the preoperative calculation was better; this was not statistically significantly different from random expectation $(50 / 50, p=0.53) .{ }^{5}$

In eyes that have undergone prior refractive surgery, it is particularly difficult to choose an IOL power given that there are post-surgical changes which are often difficult to account for with the currently available IOL formulas. An earlier study has shown that post-LASIK (laser-assisted in situ keratomileusis) eyes were less likely than normal eyes to be within $\pm 0.5 \mathrm{D}$ of emmetropia. More recently, in post-refractive surgery eyes, it has been reported that the ORA System has the highest predictive accuracy of IOL power when compared to the Haigis L formula, Shammas formula, and clinical data bases of surgeon choices. ${ }^{16,17}$

With respect to toric lenses, axis alignment requires extreme precision as 6\% of the cylindrical correction is lost for every 10 degrees of misalignment. ${ }^{18}$ Yesilirmak et al. evaluated intraoperative wavefront aberrometry-guided toric IOL placement in small cohort of postrefractive surgery eyes, and found that the ORA System has a lower mean prediction error $(0.43 \mathrm{D})$ compared to preoperative calculations using the IOLMaster ${ }^{\circledR}$ (Zeiss, Oberkochen, Germany) (0.77 D) and the American Society of Cataract and Refractive Surgery (ASCRS) calculator (0.61 D). ${ }^{19}$ Additional studies evaluating limbal relaxing incision placement have been unsuccessful in deterring the superiority of intraoperative versus preoperative planning. ${ }^{16}$

Extraneous factors such as eyelid pressure, eyelid speculums used in the pre- and intraoperative setting, and post-surgical changes can all add an element of variability with respect to astigmatism and spherical power to the overall measurements acquired during intraoperative aberrometry. Furthermore, intraoperative factors such as main and paracentesis wounds can alter the overall corneal shape and when coupled with varying levels of intraocular pressures throughout surgery, often result in vastly different astigmatic and spherical corrections. ${ }^{20,21}$

Recent studies by Yesilirmak et al. and Epitropoullos et al. have shown that the ORA System is a useful tool especially in post-refractive eyes and for toric IOLS, 19,22 and should be considered as an additional tool to help augment current methods of preoperative measurements and IOL power formulas.

\section{Conclusions}

With the added improvement in preoperative imaging and IOL formulas the utility of intraoperative imaging in correcting astigmatism and selecting IOL powers in routine cataract surgery, is an adjuvant to current methods, especially in post-refractive surgery eyes and toric IOL implantations. Though studies have shown effectiveness of utilising intraoperative imaging in cataract surgery, larger retrospective studies comparing the accuracy of current IOL formulas versus intraoperative aberrometry in both normal and post-refractive surgery eyes are needed to establish an industry standard. $\square$
Lee AC, Qazi MA, Pepose JS. Biometry and intraocular lens power calculation. Curr Opin Ophthalmol. 2008;19:13-7. Zhang $Y$, Llang XY, Lu S, et al. Accuracy of intraocular tens power calculation formulas for highly myopic eyes. Journal of Ophthalmology. 2016,2016.1-7.

Piometry. Curr Opin Ophthalmol. 2012;23:47-53

4. Gale RP, Saldana M, Johnston RL, et al. Benchmark standards for refractive outcomes after NHS cataract surgery. Eye (Lond). 2007:23:149-52.
Davison JA, Potvin R. Preoperative measurement vs intraoperative aberrometry for the selection of intraocular lens sphere power in normal eyes. Clin Ophthalmol. 2017;11:923-9. Titiyal JS, Kaur M, Jose C, et al. Comparative evaluation of toric intraocular lens alignment and visual quality with marking Wajer WJ, Kreutzer T, Dirisamer M, et al. Comparison of visua 2 methe implantation. J Cataract Refract Surg. 2017;43:1281-6. Webers VSC, Bauer NJC, Visser N, et al. Image-guided system versus manual marking for toric intraocular lens alignment in versus manual marking for toric intraocular lens alignment Elhofi AH, Helaly HA. Comparison between digital and manual marking for toric intraocular lenses. Medicine. 2015;94:e1618-4.

0. Hura AS, Osher RH. Comparing the Zeiss Callisto eye and the Alcon Verion image guided system Toric lens alignment technologies. J Refract Surg. 2017;33:482-7. 
11. Mehravaran $S$, Asgari S, Bigdeli S, et al. Keratometry with five different techniques: a study of device repeatability and inter-device agreement. Int Ophthalmol.

12. Behshad S, Tucker J, Garg SS. Toric intraocular Iens alignment: manual versus automated alignment techniques for Toric IOLS. Int Ophthalmol Clin. 2016;56:71-84.

13. Montes de Oca I, Kim EJ, Wang L, et al. Accuracy of toric intraocular lens axis alignment using a 3-dimensional computer-guided visualization system. I Cataract Refract Surg. 2016;42:550-5.

14. Zhou F, Jiang W, Lin Z, et al. Comparative meta-analysis of toric intraocular lens alignment accuracy in cataract patien mage-guided system versus manual marking. J Cataract
Refract Surg. 2019;45:1340-5.

5. Packer M. Effect of intraoperative aberio postoperative enhancement: Retrospective study. J Cataract Refract Surg. 2010;36:747-55.

16. Seamont DA. Intraoperative aberrometry, 2019. Available at: https://eyewiki.aao.org/Intraoperative_aberrometry (accessed 9 September 2019)

17. lanchulev T, Hoffer $\mathrm{KJ}, \mathrm{YoO} \mathrm{SH}$, et al. Intraoperative refractive biometry for predicting intraocular lens power calculation after prior myopic refractive surgery. Ophthalmology. 2014; $121: 56-60$

18. Tognetto D, Perrotta AA, Bauci F, et al. Quality of images with toric intraocular lenses. J Cataract Refract Surg. 2018;44:376-81
19. Yesilirmak N, Palioura S, Culbertson W, et al. Intraoperative wavefront aberrometry for Toric intraocular lens placement in eyes with a history of refractive surgery. I Refract Surg. 2016;32:69-70

20. Stringham J, Pettey J, Olson RJ. Evaluation of variables affecting intraoperative aberrometry. I Cataract Refract Surg.

2012;38:470-4.
21. Huelle JO, Katz T, Druchkiv V, et al. First clinicial results on the feasibility, quality and reproducibility of aberrometry based intraoperative refraction during cataract surgery Br J Ophthalmol. 2014;98:1484-91.

22. Epitropoulos AT. Visual and refractive outcomes of a toric presbyopia-correcting intraocular lens. J Ophthalmol. 2016;2016:1-6. 